\title{
Management of patients with acute leukemia during the COVID-19 outbreak: practical guidelines from the acute leukemia working party of the European Society for Blood and Marrow Transplantation
}

\author{
Eolia Brissot $\mathbb{D}^{1} \cdot$ Myriam Labopin ${ }^{1} \cdot$ Frédéric Baron $\mathbb{1}^{2} \cdot$ Ali Bazarbachi ${ }^{3} \cdot$ Gesine Bug ${ }^{4} \cdot$ Fabio Ciceri ${ }^{5,6}$. \\ Jordi Esteve $\mathbb{1}^{7}$. Sebastian Giebel ${ }^{8}$ - Maria H. Gilleece $\mathbb{1}^{9} \cdot$ Norbert-Claude Gorin $^{10}$ • Francesco Lanza ${ }^{11}$. \\ Zinaida Peric $^{12} \cdot$ Annalisa Ruggeri $^{5} \cdot$ Jaime Sanz $\mathbb{D}^{13} \cdot$ Bipin N. Savani $\mathbb{D}^{14} \cdot$ Christoph Schmid $^{15} \cdot$ Roni Shouval $^{16}$. \\ Alexandros Spyridonidis ${ }^{17} \cdot$ Jurjen Versluis $^{18} \cdot$ Arnon Nagler $^{19} \cdot$ Mohamad Mohty $^{1}$
}

Received: 15 May 2020 / Revised: 26 May 2020 / Accepted: 3 June 2020 / Published online: 11 June 2020

(c) Springer Nature Limited 2020

Severe acute respiratory syndrome coronavirus-2 (SARSCoV-2) is the novel coronavirus first detected in Wuhan, China. It causes an infection named coronavirus disease 2019 (COVID-19), and is now spreading worldwide. Currently, there are no approved treatment options in Europe and there is no available vaccine. Reports from China, Europe, and USA suggest a high mortality rate and stretched intensive care unit (ICU) capacity [1-3]. It has been found that the main risk factors for death are obesity, hypertension, diabetes, male gender, and older age. Moreover, Liang et al. reported that $1 \%$ of COVID-19 patients had a history of cancer, higher than that of the overall Chinese population $(0.29 \%)$, with lung cancer being the

Eolia Brissot

eolia.brissot@aphp.fr

1 Saint-Antoine Hospital, Sorbonne University, AP-HP, INSERM UMRs 938, Paris, France

2 Department of Hematology, University of Liège, Liège, Belgium

3 Department of Internal Medicine, American University of Beirut Medical Center, Beirut, Lebanon

4 University Hospital, Goethe University Frankfurt, Frankfurt am Main, Germany

5 Haematology and Bone Marrow Transplant Unit, IRCCS San Raffaele Scientific Institute, Milan, Italy

6 Università Vita-Salute San Raffaele, Milan, Italy

7 Hematology Department, Hospital Clinic, Barcelona, Spain

8 Department of Bone Marrow Transplantation and Oncohematology, Maria Sklodowska-Curie Institute, Oncology Center, Gliwice, Poland

9 Yorkshire Blood and Marrow Transplant Programme, Haematology Department, St James's Institute of Oncology, most frequently found. Moreover, patients with cancer seemed to have a higher risk of severe events (ICU admission, need of assisted ventilation, death), reaching $39 \%$ versus $8 \%$ in those not suffering from a neoplasia [4]. Their study suggests that hospital admission and recurrent outpatient visits, inherent to cancer patients' management, are potential risk factors for SARS-CoV-2 infection. Another aspect which may probably be even more relevant in cancer patients is their inability to receive the necessary care in time, under the changed scenario of a viral pandemic management.

Scarce data are available on patients with hematological malignancies. Although some recommendations for

Leeds, UK

10 European Society for Blood and Marrow Transplantation, Hopital Saint-Antoine, Paris, France

11 Hematology Unit, Romagna Transplant Network, Ravenna, Italy

12 Bone Marrow Transplant Unit, University Hospital Center Rebro, Zagreb, Croatia

13 University Hospital La Fe, Valencia, Spain

14 Department of Hematology-Oncology, Vanderbilt University Medical Center, Nashville, USA

15 Klinikum Augsburg, II Medizinische Klinik, Augsburg, Germany

16 Adult Bone Marrow Transplantation Service, Memorial Sloan Kettering Cancer Center, New York, NY, USA

17 BMT Unit, University Hospital of Patras, Patras, Greece

18 Department of Hematology, Erasmus MC Cancer Institute, University Medical Center, Rotterdam, The Netherlands

19 Chaim Sheba Medical Center, Tel Aviv University, TelHashomer, Israel 
Table 1 Recommendation for management of patients with acute leukemia during the COVID-19 outbreak.

\begin{abstract}
General points
- Screening for COVID-19 infection before initiating chemotherapy.

- Cytogenetics and molecular biology should be awaited before starting treatment.

- MRD molecular remission should be useful to consider omitting one cycle of consolidation.

- Outpatient visits should be as much as possible deferred or substituted with telemedicine visites.

AML Patient FIT for Favorable and intermediate Induction: "3+7" should be intensive therapy cytogenetics risk considered

Consolidation: cytarabine should be reduced to $1.5 \mathrm{mg} / \mathrm{m}^{2}$

Adverse cytogenetic risk Consider if real chance of going to allo-HSCT exists

Patient UNFIT for Azacytidine or low dose of cytarabine in monotherapy, intensive therapy hydroxycarbamide, palliative care

ALL Maintaining recommended dose of glucocorticoids, especially during prephase, induction and consolidation.

Allo- $\quad$ Discuss indication for allo-HCT on case-by-case.

HSCT Nonurgent allo-HCT should be deferred as much as possible.

High-risk allo-HCT such as for refractory AL or patient with a high risk of NRM should not proceed.
\end{abstract}

$A L L$ acute lymphoblastic leukemia, Allo-HSCT allogeneic stem cell transplantation, $A M L$ acute myeloid leukemia, MRD measurable/minimal residual disease, " $3+7$ " daunorubicin and cytarabine.

management of those with solid cancer and hematologic neoplasms have been published, more practical guidelines may be required for individual types of malignancies [5]. Particularly acute leukemia (AL) patients who suffer from a profound and long-lasting humoral and cellular immune deficiency [6], may benefit from tailored recommendations. Moreover, AL patients under intensive chemotherapy are at a high risk of requiring ICU care, and of mortality in case of complications [7]. Therefore, special attention should be paid to this specific population during the ongoing COVID19 outbreak. Here, we summarize some recommendations to help AL patients' management in the current situation. The aims are to minimize the requirement for ICU admission, without compromising the patient's chance of being adequately treated.

\section{General principle}

Patients should be aware of their frailty to COVID-19 infection and specific hygiene measures and importance of social distancing should be explained.

All journeys outside the hematological department should be discussed and adapted for the situation. For example, it should be ensured that outpatient transportation services bring patients to the scheduled time of their consultation, in order to minimize the time spent in the waiting area. For those requiring oral or subcutaneous treatment administration, one needs to minimize hospital visits. Possible strategies for this are offering telephone or video consultations, cutting nonessential face-to-face follow-up, using home-delivery services for medicines, and using local services for blood tests.

\section{Screening}

Given that percentage of asymptomatic cases of COVID-19 can be high [8], all newly diagnosed patients with acute myeloid leukemia (AML) or acute lymphoblastic leukemia (ALL) should be screened for COVID-19 infection by PCR and high-resolution thoracic computerized tomography scan before initiating chemotherapy [9] (Table 1). The screening should be repeated before each course of chemotherapy. If positive, the decision to initiate or continue the treatment needs to made on a case-by-case basis.

\section{Clinical research}

In terms of clinical research, most clinical trials have been suspended during the pandemic. However, in some cases, clinical trials allow access to off-label drugs or combinations which can be highly beneficial. For patients already included in clinical trials, their participation should, in principle, continue. Nevertheless, the patients' safety must remain the priority and, similarly to the recommendation for those treated outside clinical trials, outpatient visits must be replaced by e-health assessment whenever possible. Furthermore, for those receiving oral treatment in the frame of a clinical trial, most clinical research organizations now organize home delivery of the investigated medication to 
avoid hospital visits. Alternatively, hospital pharmacies should be authorized to deliver 2 or 3 months' worth of medication, rather than the standard one. Whenever possible, follow-up by e-health assessment should be preferred to avoid hospital visits.

\section{Acute myeloid leukemia}

\section{Patients fit to receive intensive therapy}

For patients with favorable or intermediate risk acute myeloid leukemia (AML) [10] who are fit to receive intensive chemotherapy, the standard " $3+7$ " induction should be considered [11]. For AML with FLT3ITD mutation, midostaurin may be added to induction and consolidation as it prolongs OS and EFS [12].

For consolidation, the dose of cytarabine could be reduced to $1.5 \mathrm{~g} / \mathrm{m}^{2}$ instead of $3 \mathrm{~g} / \mathrm{m}^{2}$ for all patients. Indeed, prospective studies showed that consolidation, in association with anthracycline, with either intermediate or high dose of cytarabine, did not result in significant differences in the 5-year overall survival, whereas prolongation of neutropenia and higher transfusion demands were observed in the high dose cytarabine arm [13-15]. The use of G-CSF should be recommended after each cycle to reduce the duration of neutropenia. In patients who have negative measurable/minimal residual disease (MRD) after two cycles of chemotherapy, omission of the fourth cycle of consolidation should be discussed. In this case, the MRD should be very closely monitored, and maintenance therapy considered, especially in those cases.

Patients with an adverse cytogenetic risk should receive intensive therapy if a real chance of going to allogeneic stem cell transplantation exists.

In the case of acute promyelocyte leukemia (APL), chemotherapy should be initiated without delay. Patients with standard-risk APL (white blood cells $<10 \times 10^{9} / \mathrm{L}$ ) should receive all-trans-retinoic acid (ATRA) and arsenic trioxide (ATO) as frontline following the standard guideline for APL management (i.e., avoidance of G-CSF for the risk of differentiation syndrome). For high-risk APL, induction should be performed with idarubicine, ATRA, and ATO.

\section{Patients unfit to receive intensive therapy}

Newly diagnosed patients with AML, who are unfit for intensive treatment, hypomethylating agents (HMA) or lowdose cytarabine monotherapy (LDAC) could be given in the case of no-proliferative disease. The addition of venetoclax should be discussed on a case-by-case basis, considering the positive impact on $\mathrm{CR}$ rate and $\mathrm{OS}$ in combination with HMA or LDAC, but also the risk of tumor lysis syndrome and myelosuppression (https://www.fda.gov/drugs/fda-a pproves-venetoclax-combination-aml-adults). After the first cycle with this combination, if medullary blast infiltration is $<5 \%$, dose adjustments, duration of venetoclax, and/or the use of G-CSF are recommended to avoid prolonged cytopenia. Taking into account age, comorbidities, and disease characteristics, patients could also be managed with supportive care and, possibly, eventually hydroxycarbamide.

For patients with relapsed or refractory AML, each team should carefully assess the risks and benefits of pursuing a curative approach on a case-by-case basis. Molecular targeted therapy (e.g., enasidenib, ivosidenib, sorafenib, gilteritinib, etc.) should be discussed, considering the rate of complete remission and the duration of the response that can be expected, with a view to postponing an intensive treatment or allogeneic hematopoietic cell transplantation (allo-HCT).

\section{Acute lymphoblastic leukemia}

In ALL, one major question is the use of glucocorticoids, as they remain essential components of ALL therapy. They appeared to be effective in reducing immunopathological damage [16], but there are concerns about their possible promotion of viral rebound and adverse events. Taking into account the major role of glucocorticoids in the treatment of ALL, and the paucity of information on their potential negative role in COVID-19 infections, physicians should use the recommended dose of glucocorticoids, especially during, the prephase, induction, and consolidation, with a major concern on preventing bacterial and fungal infections. The use of asparaginase should be carefully monitored considering the inherent risk of thrombotic complications of this drug, especially knowing that COVID-19 can lead to systemic coagulation disorders, and thrombotic complications. The use of blinatumomab or inotuzumab should not be delayed as their benefit in terms of survival has been established.

For Philadelphia-chromosome positive ALL, inhibitors of tyrosine kinase should be maintained considering their positive impact on OS and EFS.

\section{Stem cell transplantation}

The EBMT recommendations for management of allo-HCT during the COVID-19 outbreak have been recently published [17]. Nonurgent allo-HCT procedures should be deferred as much as possible. Due to the rapidly changing situation, access to a stem cell donor may be restricted by the fact that the donor may become infected at the harvest centers in the middle of a strained health care system, or by 
travel restrictions across international borders. It is, therefore, strongly recommended to have secured stem cell product access, by cryopreserving the product before the start of conditioning. In situations when this is not possible, an alternative back-up donor should be identified. The impact of COVID-19 on a timely graft availability, on cellular therapy unit organization, and on ICU capacity should be considered for each patient with allo-HCT indication. It is necessary to highlight the yet unknown impact of COVID-19 infection on outcomes, when counseling patients on the benefits and risks of the allo-HCT procedure.

All patients who have a high risk of disease progression without allo-HCT should still be considered candidates for the procedure according to standard clinical practice. More controversial allo-HCT indications such as refractory AL, or patients with a high risk of non-relapse mortality should be avoided.

Overall, the management of patients with $\mathrm{AL}$ in the COVID-19 outbreak is a major challenge, as this hematological malignancy requires rapid treatment, which may result in a requirement for admission to an ICU unit. Physicians should therefore carefully balance the risk of COVID-19 infection itself against the benefit of antileukemic intensive treatment on a case-by-case basis, within the individual resources of each medical institution.

Acknowledgements We acknowledge all colleagues for helpful discussions, which allowed to refine the content of this manuscript. We thank Pr. J.V. de Melo (University of Adelaide, Australia) for critical reading of this manuscript.

Author contributions All authors contributed to the conception, writing, critical review, and final approval of the manuscript.

\section{Compliance with ethical standards}

Conflict of interest The authors declare that they have no conflict of interest.

Publisher's note Springer Nature remains neutral with regard to jurisdictional claims in published maps and institutional affiliations.

\section{References}

1. Grasselli G, Pesenti A, Cecconi M. Critical care utilization for the COVID-19 outbreak in Lombardy, Italy: early experience and forecast during an emergency response. JAMA. 2020;323:1545-46.

2. Yang X, Yu Y, Xu J, Shu H, Xia J, Liu H, et al. Clinical course and outcomes of critically ill patients with SARS-CoV-2 pneumonia in Wuhan, China: a single-centered, retrospective, observational study. Lancet Respir Med. 2020;8:475-81.

3. Bhatraju PK, Ghassemieh BJ, Nichols M, Kim R, Jerome KR, Nalla AK, et al. Covid-19 in critically ill patients in the Seattle region-case series. N Engl J Med. 2020;382:2012-22.

4. Liang W, Guan W, Chen R, Wang W, Li J, Xu K, et al. Cancer patients in SARS-CoV-2 infection: a nationwide analysis in China. Lancet Oncol. 2020;21:335-7.

5. Malard F, Mohty M. Management of patients with multiple myeloma during the COVID-19 outbreak. Lancet Haematol. 2020;7:e435-37.

6. Portugal RD, Garnica M, Nucci M. Index to predict invasive mold infection in high-risk neutropenic patients based on the area over the neutrophil curve. J Clin Oncol. 2009;27:3849-54.

7. Halpern AB, Culakova E, Walter RB, Lyman GH. Association of risk factors, mortality, and care costs of adults with acute myeloid leukemia with admission to the intensive care unit. JAMA Oncol. 2017;3:374-81.

8. Mizumoto K, Kagaya K, Zarebski A, Chowell G. Estimating the asymptomatic proportion of coronavirus disease 2019 (COVID19) cases on board the Diamond Princess cruise ship, Yokohama, Japan, 2020. Euro Surveill. 2020;25:2000180.

9. Ai T, Yang Z, Hou H, Zhan C, Chen C, Lv W, et al. Correlation of chest CT and RT-PCR testing in coronavirus disease 2019 (COVID-19) in China: a report of 1014 cases. Radiology. 2020:200642. In press.

10. Dohner H, Estey E, Grimwade D, Amadori S, Appelbaum FR, Buchner T, et al. Diagnosis and management of AML in adults: 2017 ELN recommendations from an international expert panel. Blood. 2017;129:424-47.

11. Lowenberg B, Pabst T, Vellenga E, van Putten W, Schouten HC, Graux C, et al. Cytarabine dose for acute myeloid leukemia. N Engl J Med. 2011;364:1027-36.

12. Stone RM, Larson RA, Dohner H. Midostaurin in FLT3-mutated acute myeloid leukemia. N Engl J Med. 2017;377:1903.

13. Schaich M, Rollig C, Soucek S, Kramer M, Thiede C, Mohr B, et al. Cytarabine dose of $36 \mathrm{~g} / \mathrm{m}(2)$ compared with $12 \mathrm{~g} / \mathrm{m}(2)$ within first consolidation in acute myeloid leukemia: results of patients enrolled onto the prospective randomized AML96 study. J Clin Oncol. 2011;29:2696-702.

14. Burnett AK, Hills RK, Milligan DW, Goldstone AH, Prentice AG, McMullin MF, et al. Attempts to optimize induction and consolidation treatment in acute myeloid leukemia: results of the MRC AML12 trial. J Clin Oncol. 2010;28:586-95.

15. Bradstock KF, Matthews JP, Lowenthal RM, Baxter H, Catalano $\mathrm{J}$, Brighton $\mathrm{T}$, et al. A randomized trial of high-versus conventional-dose cytarabine in consolidation chemotherapy for adult de novo acute myeloid leukemia in first remission after induction therapy containing high-dose cytarabine. Blood. 2005;105:481-8.

16. Cinatl J Jr., Michaelis M, Morgenstern B, Doerr HW. High-dose hydrocortisone reduces expression of the pro-inflammatory chemokines CXCL8 and CXCL10 in SARS coronavirus-infected intestinal cells. Int J Mol Med. 2005;15:323-7.

17. Ljungman P. The challenge of COVID-19 and hematopoietic cell transplantation; EBMT recommendations for management of hematopoietic cell transplant recipients, their donors, and patients undergoing CAR T-cell therapy. Bone Marrow Transplant. 2020, In press. 